\title{
High dose of botulinum toxin type-A (BTX/A): safety and efficacy in patients with cerebral palsy
}

\author{
Yasser Awaad ${ }^{1}$, Hassan Tayem ${ }^{1}$, Sharon Munoz ${ }^{1}$, Ronald Thomas ${ }^{2}$, \\ Sherif Soliman ${ }^{3}$, Anne Marie Michon ${ }^{1}$, Sarah Minarik ${ }^{4}$ \\ ${ }^{1}$ Neurology Division, and ${ }^{2}$ Children's Research Center of Michigan, Children's Hospital of Michigan, \\ Wayne State University, School of Medicine, Detroit, Michigan, U.S.A. \\ ${ }^{3}$ Wayne State University, School of Medicine, Detroit, Michigan, U.S.A. \\ ${ }^{4}$ Oakwood Health Care System Dearborn, Michigan, U.S.A.
}

\begin{abstract}
Cerebral palsy (CP) is a severely debilitating condition. The underlying insult results in irreversible damage to the central nervous system, treatment is essentially palliative. Botulinum toxin type-A (BTX/A) Botox ${ }^{\circledR}$ Allergan, Inc. injections have been found to be effective palliation for dynamic spasticity in CP. There is a paucity of data regarding the maximum dose tolerated in the pediatric population. The present study examines the safety and efficacy of BTX/A therapy at up to $40 \mathrm{U} / \mathrm{kg}$. Twenty-two patients were treated from 1995 to 1999. The population included four adults and 18 pediatric patients. Patients were in three groups: diplegia $(n=12)$, hemiplegia $(n=5)$, and quadriplegia $(n=5)$. They were evaluated by the Modified Ashworth Scale. The incidence and severity of complications is also reported. The significance of these data is also commented upon. This study suggests that BTX/A therapy is safe, efficacious, and cost effective at higher doses, and should be considered as part of a comprehensive therapeutic regimen in appropriate candidates. (J Pediatr Neurol 2004; 2(2): 91-96).
\end{abstract}

Key words: botulinum toxin, cerebral palsy, spasticity, safety and efficacy.

Correspondence: Yasser Awaad, M.D., MSc

Associate Professor of Pediatrics,

Director of Pediatric Neurology and Movement Disorders,

Oakwood Healthcare System, University of Michigan, U.S.A.

Office Address: 21031 Michigan Ave., Dearborn,

MI 48124, U.S.A.

Phone: (313) 791-4323, fax: (313) 791-4325.

E-mail: AwaadY@oakwood.org

Received: February 17, 2004.

Revised: March 16, 2004

Accepted: March 29, 2004.

\section{Introduction}

Over 500,000 patients in the United States are afflicted with cerebral palsy (CP), with an incidence of 5000-7000 new cases per year (1). These patients suffer from painful spasticity, which interferes with gait, joint positioning, and hygiene. CP results from a variety of central nervous system lesions of various etiologies. One of the goals of current clinical management is to reduce the painful and debilitating spasticity that accompanies CP as well as improving the activities of daily living. Since the subsequent cortical insult is irreversible. Current strategies include oral medications, tendonoplasty, physical therapy, posterior rhizotomy, neuromuscular blockade, and intrathecal baclofen therapy (2-4).

Botulinum toxin-type A (BTX/A), the most potent neurotoxin known, is produced by the gram positive bacteria Clostridium botulinum. It is believed to block neuromuscular transmission by inhibiting the release of acetylcholine at presynaptic cholinergic terminals by enzymatically destroying synaptosomal associated protein 25 (5). These effects are readily reversible due to axonal sprouting. Furthermore, it appears that there is no substantial residual atrophy after multiple BTX/ A injections, although this subject is still under rigorous investigation (6).

BTX/A was first used therapeutically to treat strabismus and blepharospasm. However, it has since proven to be a safe and effective treatment for a variety of neuromuscular disorders. It has recently been used as a treatment for CP (1,7-11), although its application to pediatric patients has been limited by the lack of experience with its use in this population. Several studies generally concur that BTX/A injections are potentially beneficial to patients with $\mathrm{CP}$ related spasticity; many questions remain however regarding the safety of multiple injections and the maximum tolerated dose in pediatric patients. There is very little data 
Table 1. Demographic characteristics of the patients

\begin{tabular}{|c|c|c|c|c|c|c|}
\hline Groups & $\begin{array}{c}\text { Numbers } \\
\mathbf{n}\end{array}$ & $\begin{array}{c}\text { Average age } \\
\text { (years) }\end{array}$ & $\begin{array}{c}\text { Males } \\
\mathbf{n}\end{array}$ & $\begin{array}{l}\text { Females } \\
\mathbf{n}\end{array}$ & $\begin{array}{c}\text { Ambulatory } \\
\mathbf{n}\end{array}$ & $\begin{array}{l}\text { Non-ambulatory } \\
\mathbf{n}\end{array}$ \\
\hline Diplegia & 12 & 9 & 7 & 5 & 8 & 4 \\
\hline Hemiplegia & 5 & 6 & 2 & 3 & 5 & \\
\hline Quadriplegia & 5 & 1 & 6 & 3 & 2 & 5 \\
\hline Total & 22 & 9 & 12 & 10 & 13 & 9 \\
\hline
\end{tabular}

Table 2. Units per $\mathrm{kg}$ and weeks of sustained improvement after injection using higher dosages

\begin{tabular}{llllllllllllllllllllllllllll}
\hline \hline Patient no & 1 & 2 & 3 & 4 & 5 & 6 & 7 & 8 & 9 & 10 & 11 & 12 & 13 & 14 & 15 & 16 & 17 & 18 & 19 & 20 & 21 & 22 \\
Weight/kg & 32 & 19 & 12 & 52 & 35 & 28 & 15 & 26 & 12 & 18 & 13 & 16 & 55 & 27 & 58 & 8 & 22 & 13 & 33 & 27 & 15 & 20 \\
Dose/unit & 1000 & 400 & 400 & 800 & 1000 & 400 & 500 & 800 & 300 & 400 & 300 & 500 & 1200 & 500 & 1300 & 300 & 800 & 500 & 1200 & 800 & 300 & 700 \\
Unit/kg & 31.4 & 21 & 34 & 15 & 29 & 14 & 33 & 30 & 24 & 22.5 & 23.4 & 31 & 22 & 19 & 22 & 38 & 37 & 40 & 37 & 30 & 21 & 35.5 \\
$\begin{array}{l}\text { Improvement/ } \\
\text { weeks }\end{array}$ & 30 & 40 & 17 & 13 & 33 & 33 & 29 & 39 & 15 & 17 & 61 & 69 & 26 & 37 & 25 & 42 & 14 & 31 & 14 & 32 & 28 & 30 \\
\hline \hline
\end{tabular}

The above indicated the maximal dose utilized in the individual patients.

available regarding these issues. Therefore, many investigators have made the implicit assumption that pediatric tolerance is equivalent to adult tolerance.

Many of the initial indications for BTX/A therapy require relatively low doses; therefore, it has not been necessary to explore the safety of higher doses. However, new indications such as $\mathrm{CP}$ may require higher doses in order to achieve maximal benefit from the therapy and longer duration of relief. Up to $6 \mathrm{U} / \mathrm{kg}$ have been used in adults without systemic side effects (9) and, in children with $\mathrm{CP}, 8-10 \mathrm{U} / \mathrm{kg}$ have been successfully utilized (10). These doses unfortunately, are not sufficient to provide relief for many patients who suffer severe debilitating spasticity. There has been little data regarding the efficacy and safety of BTX/A therapy in pediatric patients for whom traditional doses are insufficient. The present study investigates the efficacy and safety of repeated BTX/A injections at doses of up to $40 \mathrm{U} / \mathrm{kg}$ in pediatric and young adult patients suffering from spasticity of various etiologies. In patients who were nonresponsive to low doses, the dose was gradually increased while therapeutic benefits and potential adverse reactions were closely monitored. Special consideration is given to the issue of BTX/A safety at therapeutic doses. We are reporting the effects of continued BTX/A treatment in patients over a four year study period.

\section{Materials and Methods}

The study included a total of $22 \mathrm{CP}$ patients from the movement disorders clinic at Children's Hospital of Michigan. After carefully counseling the patients and their families regarding the risks and benefits of therapy, informed consent was obtained from patient or legal guardian prior to initiation of therapy. Patient demographic data is shown in Table
1. Patients were given an initial dose of 10-12 U/ $\mathrm{kg}$ of botulinum toxin type-A: $10.0 \mathrm{U} / 0.1$ dilution (CAllergan, Inc.) and later transferred to a higher dose (Table 2) if they did not experience clinically significant improvement in their spasticity, as indicated by functional gains (such as gait improvement in both distance and time, transfering, better fitting of orthodics and seating, improvement in range of motion (ROM), and hygiene both bathing and toileting; the aforementioned has been reported by patient/caregiver self report questionnaire) and an improved Ashworth score.

Our inclusion criteria for the study were as follows:

1. The presence of spasticity refractory to oral medication or other treatment modalities such as alcohol or phenol block; or, the patient was intolerant of other therapies.

2. An initial average Modified Ashworth Scale (MAS) score of three or greater in the upper extremities was obtained by assessing wrist flexion and extension, elbow flexion and extension and lower extremities by assessing hip adduction, abduction, and flexion, knee flexion and extension, and planter flexion.

3. The absence of contraindications to BTX/ A therapy, which include fixed contractures, aminoglycoside therapy, pregnancy, and myasthenia gravis or other neuromuscular disease.

The presence of one or more of the following constituted criteria for the discontinuation of therapy:

1. The presence of clinically significant weakness (inability to walk, significant decrease in deep tendon reflexes in ambulatory patients, loss of head control, and frog leg position in non-ambulatory patients), dysphagia, or respiratory depression.

2.The patient remained unresponsive to therapy for 


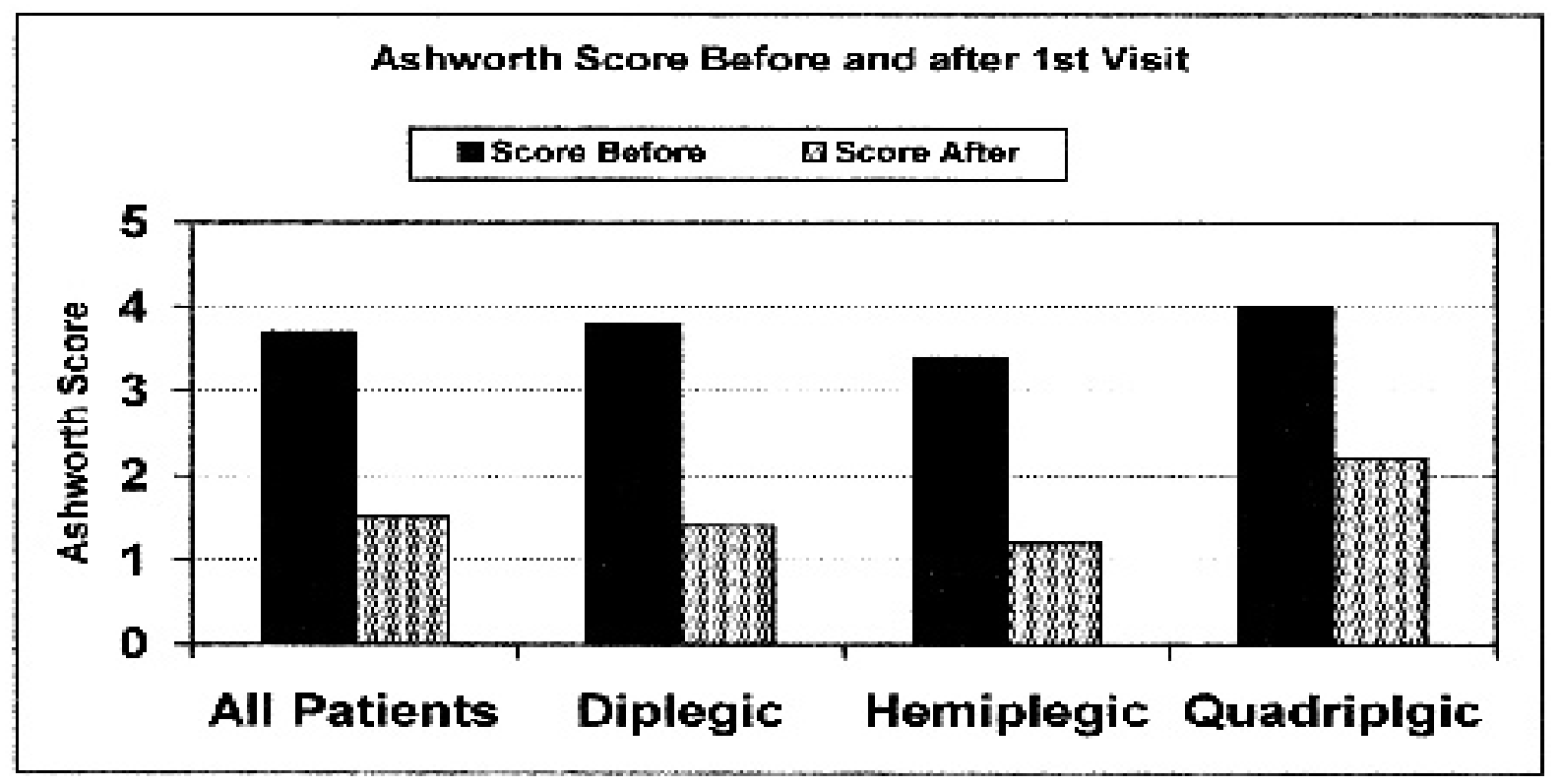

Figure 1. Ashworth score before and after 1st visit.

six months after the maximum dose was attained.

3. The patient developed antibodies to BTX/A and became unresponsive to therapy (antibody formation was only assessed in patients who became unresponsive).

4. The presence or development of contraindications to BTX/A therapy.

5. Patient or guardian request to discontinue therapy.

All patients underwent a preinjection screening and videotaping. The preinjection screening process included a complete neurological exam by a pediatric neurologist (YA), as well as an evaluation by a physical therapist. The neurological exam included an extensive history. The physical therapy evaluation included evaluation of spasticity using the Modified Ashworth Scale (MAS) (12). After determining patient suitability and obtaining informed consent, BTX/A therapy was initiated. Intramuscular injections were administered without sedation or local anesthesia. Each muscle was infiltrated at multiple sites, using a 27-gauge electromyography (EMG) needle under EMG guidance. The injection sites were in the bulk of the muscle near the neuromuscular junctions to maximize therapeutic effects. A follow up examination was conducted two weeks after the initial visit to assess the safety and efficacy of the therapy. Patients were carefully monitored for adverse reactions following each injection. Patients initially remained in clinic for 12 hours post injection to monitor immediate adverse reactions; however, this practice was discontinued as increasing clinical experience indicated such reactions were highly unlikely. Caregivers were instructed to report adverse reactions immediately. The caregivers were called 3, 5 and 7 days after the injection to check patient status. All patients were enrolled in aggressive physical therapy/occupational therapy (PT/OT) as well as utizing orthodics and undergoing serial casting as needed as an adjuvant to BTX/A treatment. Pre and post therapy MAS scores were obtained (Figure 1).

Efficacy was assessed using both objective and subjective criteria. Patients or caregivers, during follow up, rated the percent improvement during the course of therapy. They were instructed to base the score upon several criteria including changes in pain, spasticity, positioning, mobility, and sleep. Similar results were noted in other studies using the Pediatric Evaluation of Disabalitiy Inventory (PEDI) (13). This serves as a subjective measure of patient response to therapy, while MAS scores are an objective one. Patients/caregivers were instructed to schedule an appointment for the next visit when improvement diminished to approximately 30\% above baseline, usually 6-9 months after the prior visit. Table 2 shows weeks of sustained improvement after the first injection.

Data analyses conducted with this sample were descriptive in nature. Therefore, measures of central tendency (mean, median, and mode) and variability (standard deviation, minimum, and maximum values) were generated and examined using SPSS version 10 . Where variables were discontinuous in scale (nominal or ordinal) either the mode and/or median is reported to describe the appropriate measure of central tendency. Where variables were continuous in scale (interval or ratio) the mean and standard deviation are reported. Presentation of the distribution of responses and the summarization of the study variables in this manner provide us with the basis for more complex analyses, and the ability to explore possible relationships between different dose response categories, in future studies. 
Table 3. Clinical response to therapy

\begin{tabular}{llllccc}
\hline \hline Groups & $\begin{array}{l}\text { Average } \\
\text { dose/visit } \\
\text { (U/kg) }\end{array}$ & $\begin{array}{l}\text { Average } \\
\text { MAS before } \\
\text { treatment } \\
\text { (mean } \pm \text { SD) }\end{array}$ & $\begin{array}{l}\text { Average } \\
\text { MAS after } \\
\text { treatment } \\
\text { (mean } \pm \text { SD) }\end{array}$ & Differences & $\begin{array}{c}\text { Mean interval } \\
\text { between visits } \\
\text { (weeks) }\end{array}$ & $\begin{array}{l}\text { Mean subjective } \\
\text { improvement } \\
\text { (\%) }\end{array}$ \\
\hline Diplegia & 27.5 & $3.75 \pm 0.49$ & $1.42 \pm 0.46$ & 2.33 & 34 & 60 \\
Hemiplegia & 22 & $3.40 \pm 0.55$ & $1.2 \pm 0.45$ & 2.2 & 34 & 45 \\
Quadriplegia & 24 & $4.0 \pm 0.0$ & $2.2 \pm 0.45$ & 1.8 & 29 & 51 \\
\hline Total & 24 & $3.73 \pm 0.46$ & $1.55 \pm 0.50$ & 2.18 & 33 & 51 \\
\hline \hline
\end{tabular}

Table 4. Profiles of adverse reactions to therapy

\begin{tabular}{llllll}
\hline \hline Diagnoses & $\begin{array}{l}\text { Age } \\
\text { (years) }\end{array}$ & $\begin{array}{l}\text { Gender } \\
\text { (U/kg) }\end{array}$ & Dose/visit & Complications & $\begin{array}{l}\text { Duration } \\
\text { (days) }\end{array}$ \\
\hline Diplegia & 10.5 & Female & 30.9 & Weakness & 14 \\
Diplegia & 2 & Female & 37.7 & Weakness, flu like symptoms & 3 \\
\hline \hline
\end{tabular}

\section{Results}

The patients were first considered as a single population and then subdivided into three groups according to diagnoses: diplegia, hemiplegia, and quadriplegia. Clinical outcomes were expressed, within each group, in terms of three parameters: objective improvement, subjective improvement, and incidence of complications. Table 1 shows the demographic characteristics of each group. It shows average age, gender, and ambulatory status. The etiology of the spasticity is cerebral insults in utero in all but one patient, whose spasticity is secondary to trauma. Clinical outcomes were assessed according to both subjective and objective criteria, as shown in Table 3. The objective criterion used to measure response to therapy is the change in MAS score over the duration of the entire course of therapy. The subjective criterion was patient and/or caregiver feedback regarding improvement, which was quantified by asking caregivers to rate the percent improvement at follow-up visits. Table 3 shows average dose $/ \mathrm{kg}$, average MAS scores before and after therapy, average number of weeks in between injections, and average subjective improvement as rated by primary caregiver. Safety is a vital consideration when evaluating any new treatment protocol. Table 4 shows the types of complications experienced during the course of treatment, the dose at which they were encountered, as well as the duration of the complication. The 22 patients in the study had a total of 62 office visits during the study period. During the 62 office visits, there were three reported complications, or $4.8 \%$ of the visits. These complications were reported by two of the 22 patients in the study, or $9.1 \%$. Both patients experienced focal muscle weakness at the site of injection, which was not debilitating. In addition, one of the patients also experienced a flu like illness. As shown in Table 4, the reported complications resolved without specific therapy. In addition, neither of the patients who experienced complications elected to discontinue therapy because of complications.

\section{Discussion}

The painful spasticity that accompanies CP can be severely debilitating. It can often interfere with gait, joint positioning, and hygiene. Unfortunately, many traditional therapies for $\mathrm{CP}$ often cannot significantly improve the quality of life for many patients without considerable trauma.

While previous indications for BTX/A therapy required relatively low doses, many $\mathrm{CP}$ patients require considerably greater doses to achieve optimal benefit. Therefore, it is necessary to reevaluate the dosage schedule in order to afford these patients the benefits of therapy. The dose injected must be sufficient to maximize therapeutic effects while minimizing side effects. Two implicit assumptions regarding dose safety are called into question by the present study. The first is that animal models accurately reflect human tolerance levels while the second is that pediatric patients will only tolerate as many $\mathrm{U} / \mathrm{kg}$ of BTX/A as their adult counterparts. Previous studies have suggested that BTX/A injections are effective in reducing spasticity in pediatric patients (1,7-11). The presents study explores the efficacy and safety of extending BTX/A therapy to those children for whom traditional doses provide suboptimal relief.

With knowing the LD-50 of BTX/A, the most critical question is that of safety of high dose BTX/A therapy in the pediatric population. This issue must be addressed with respect to both short and long 
term effects of therapy. In the short term, the most common side effect was transient weakness, which resolved in all cases. While further study is required to assess long term safety, no additional side effects developed with continual therapy during the duration of this study. Prior studies have reported a complication rate of $6 \%$ using lower doses (9). The incidence of complications per visit in the present study was $4.8 \%$ and $9.1 \%$ when calculated per patient. It is important to note, however, that all complications resolved without specific treatment, and no patients elected to discontinue therapy because of complications. Although the risk of complications may rise somewhat with increasing dose, the benefits of therapy outweigh the complications at these doses. It is also likely that the incidence and severity of complications is not a simple function of dosage, rather; the result of a complex interaction between dosage and various determinants of inherent patient sensitivity. Further study is required to shed more light upon genetic, environmental, and immunologic factors that play a role in patient sensitivity.

The possibility of systemic complications is also of utmost concern when treating any patient with BTX/A therapy. Generalized muscle weakness was recently observed in two adult patients receiving Dysport (Botulinum Toxin Type-A) doses below the maximum recommended dose (14). No such systemic effect has been previously reported, despite the use of considerably higher doses in other studies, including the present study. This also tends to suggest that the occurrence of systemic side effects is most likely a complex function of patient sensitivity, rather than a direct function of dosage. The efficacy of BTX/A therapy has been expressed using both objective and subjective criteria. Both indices suggest that clinical response has been significant.

The possible development of BTX/A resistance is a potential barrier to chronic BTX/A therapy. A recent report suggests that development of antibodies does not necessarily correlate with BTX/A resistance (10). Furthermore, antibody development is most likely not a simple function of dosage, but a more complex phenomenon related to dosage, injection frequency, and inherent patient sensitivity, among other factors. The risk of developing BTX/A resistance at therapeutic doses has not been well characterized. Therefore, further study is required to quantify the increased risk, if any, of clinically significant antibody formation. Continued monitoring of these patients will hopefully shed more light upon the mechanisms and incidence of BTX/A resistance in patients treated with high and low dose BTX/A therapy. The present study suggests that pediatric patients can tolerate considerably higher doses of BTX/A than previously thought and that higher doses may have considerable therapeutic advantages over conventional doses in the management of CP. The use of higher doses reduces the frequency of injections, which reduces patient discomfort and anxiety and increases the cost effectiveness of the treatment. Higher doses also allow the injection of multiple muscle groups and a longer period between injections, which affords the patient greater relief over a longer time span. Initially, by utilizing higher dosages the cost per visit will be higher. However, in comparison between using lower and higher doses we have found the duration of clinically sustained improvement moved from 3-4 months to 6-9 months which will ultimately lower the number of injections per year from 3-4 times to 1-2 times. We also found by using the higher doses the patients achievement in physical therapy was greater and goals of therapy were reached sooner. Ultimately, the patient will return and remain in school longer and the caregiver will not miss as much work. This will in the long run lessen the financial burden on the medical system and on the society.

Theoretically, antagonist muscles will also have more time to become stronger. These effects will most likely lead to more effective physical therapy and more functional improvements in gait, joint positioning, and hygiene. In addition, BTX/A therapy may postpone or possibly obviate the need for orthopedic surgery in some patients. The cumulative effect of these benefits is a dramatic increase in the patients' quality of life, as well as that of their families.

The use of higher doses helps extend this therapy to more severely ill patients who otherwise would not be good candidates for therapy. This study strongly suggests that higher doses of BTX/A should be explored as a safe and effective treatment option in managing pediatric and adult patients. It is, of course, critical that rigorous precautions be taken when administering this treatment. Patients receiving BTX/A should also be closely monitored for systemic side effects, since patient sensitivity varies. Thus, the importance of close cooperation among the caregiving team including the pediatric neurologist, physical therapist, patient family, and other caregivers cannot be overstated. While BTX/ A injections appear to be a promising treatment for pediatric patients, there are still many important questions to be explored. It would be interesting to continue to monitor this cohort of patients in order to examine further the long term safety and efficacy of BTX/A therapy.

\section{Conclusion}

Higher doses of BTX/A, when indicated, appear to be a safe and highly effective treatment for 
spasticity in pediatric and adult patients. Higher doses of BTX/A apparently result in greater relief for a longer time period. Furthermore, this study found no evidence that higher doses result in an increase in frequency or severity of complications. Therefore, BTX/A therapy can augment other treatments to produce a dramatic improvement in the patients' quality of life with minimal side effects. Further study is needed to monitor long term safety and efficacy of BTX therapy in this population.

\section{References}

1 Koman LA, Mooney JF 3rd, Smith B, Goodman A, Mulvaney T. Management cerebral palsy with botulinum-A toxin: preliminary investigation. J Pediatr Orthop 1993; 13: 489-495.

2 Steinbok P, Reiner AM, Beauchamp R, Armstrong RW, Cochrane DD, Kestle J. A randomized clinical trial to compare selective posterior rhizotomy plus physiotherapy with physiotherapy alone in children with spastic diplegic cerebral palsy. Dev Med Child Neurol 1997; 39: 178-184.

3 Koman LA, Mooney JF 3rd, Smith B. Neuromuscular blockade in the management of cerebral palsy. J Child Neurol 1996; 11 Suppl 1: S23-S28.

4 Albright AL. Intrathecal baclofen in cerebral palsy movement disorders. J Child Neurol 1996; 11 Suppl 1: S29-S35.

5 Cai S, Sarkar HK, Singh BR. Enhancement of the endopeptidase activity of botulinum neurotoxin by its associated protein and dithiothreitol. Biochemistry 1999; 38: 6903-6910.

6 Borodic GE, Pierce LB, Ferrante R. Therapeutic botulinum toxin: histologic effects and diffusion properties botulinum and tetanus neurotoxins In: DasGupta BR (ed). Botulinum and Tetanus Neurotoxins: Neurotransmission and Biomedical Aspects. New York: Plenum press, 1993, pp $623-$ 645.

7 Cosgrove AP, Corry IS, Grahm HK. Botulinum toxin in the management of the lower limb in cerebral palsy. Dev Med Child Neurol 1994; 36: 386-396.

8 Denislic M, Meh D. Botulinum toxin in the treatment of cerebral palsy. Neuropediatrics 1995; 26: 249252.

9 Koman LA, Mooney JF 3rd, Smith BP, Goodman A, Mulvaney T. Management of spasticity in cerebral palsy with botulinum-A toxin: report of a preliminary, randomized, double-blind trial. J Pediatr Orthop 1994; 14: 299-303.

10 Gooch JL, Sandell TV. Botulinum toxin for spasticity in children with cerebral palsy. Arch Phys Med Rehabil 1996; 77: 508-511.

11 Calderon-Gonzalez R, Calderon-Sepulveda R, Rincon-Reyes M, Garcia-Ramirez J, Mino-Arango E. Botulinum toxin A in management of cerebral palsy. Pediatr Neurol 1994; 10: 284-288.

12 Bohannon RW, Smith MB. Interrater reliability of a modified Ashworth scale of muscle spasticity. Phys Ther 1987; 67: 206-207.

13 Mall V, Kirschner J, Linder M, et al. Botulinum toxin A in children with cerebral palsy: evaluation of therapy using the Pediatric Evaluation of Disability Inventory (PEDI). J Pediatr Neurol 2003; 1: 29-34.

14. Bakheit AM, Ward CD, McLellan DL. Generalised botulism-like syndrome after intramuscular injections of botulinum toxin type A: a report of two cases. J Neurol Neurosurg Psychiatry 1997; 62: 198. 